\title{
Spectrum Auction Games for Multimedia Streaming Over Cognitive Radio Networks
}

\author{
Yan Chen, Yongle Wu, Beibei Wang, and K. J. Ray Liu
}

\begin{abstract}
Cognitive radio technologies have become a promising approach to efficiently utilize the spectrum. Although many works have been proposed recently in the area of cognitive radio for data communications, little effort has been made in contentaware multimedia applications over cognitive radio networks. In this paper, we study the multimedia streaming problem over cognitive radio networks, where there is one primary user and $N$ secondary users. The uniquely scalable and delay-sensitive characteristics of multimedia data and the resulting impact on users' viewing experiences of multimedia content are explicitly involved in the utility functions, due to which the primary user and the secondary users can seamlessly switch among different quality levels to achieve the largest utilities. Then, we formulate the spectrum allocation problem as an auction game and propose three distributively auction-based spectrum allocation schemes, which are spectrum allocation using Single object pay-as-bid Ascending Clock Auction (ACA-S), spectrum allocation using Traditional Ascending Clock Auction (ACA-T), and spectrum allocation using Alternative Ascending Clock Auction (ACA-A). We prove that all three algorithms converge in a finite number of clocks. We also prove that ACA-S and ACA-A are cheatproof while ACA-T is not. Moreover, we show that ACA-T and ACA-A can maximize the social welfare while ACA-S may not. Therefore, ACA-A is a good solution to multimedia cognitive radio networks since it can achieve maximal social welfare in a cheat-proof way. Finally, simulation results are presented to demonstrate the efficiency of the proposed algorithms.
\end{abstract}

Index Terms-Multimedia, cognitive radio networks, auction, game theory, cheat-proof, social welfare.

\section{INTRODUCTION}

W ITH the advance of communication technologies, wireless access and networking has become more and more popular, which leads to a dramatic increase in the demand for radio spectrum. This phenomenon causes a critical challenge to the conventional "Command-and-Control" spectrum usage model, in which allowable spectrum uses are limited based on regulatory judgments. To address this problem, the U.S. Federal Communications Commission (FCC) proposes to use more flexible "Exclusive Use" and "Commons" models [1]. In the "Exclusive use" model, a licensee (i.e. primary user) has exclusive and transferable flexible use rights for the spectrum. In the "Commons" model, spectrum is unlicensed and an unlimited number of unlicensed users (i.e. secondary users) can share frequencies with usage rights governed by technical standards. In both models, the key issue is how to fairly, adaptively, and efficiently utilize the spectrum.

Paper approved by R. Fantacci, the Editor for Wireless Networks and Systems of the IEEE Communications Society. Manuscript received September 2, 2009; revised November 4, 2009 and February 6, 2010.

The authors are with the Department of Electrical and Computer Engineering, University of Maryland, College Park, MD 20742 USA (e-mail: \{yan, wuyl, bebewang, kjrliu\}@umd.edu).

Digital Object Identifier 10.1110/TCOMM.2010.08.090528
Cognitive radio is a technology that can enable the wireless devices to dynamically access the spectrum [2]. In the literature, researchers have proposed various approaches to optimally share the spectrum using cognitive radio technologies in different scenarios. The authors in [3] proposed to use local bargaining to achieve distributed conflict-free spectrum assignment while those in [4] formulated the spectrum access problem as a noncooperative game and proposed a learning-based distributed algorithm to obtain the correlated equilibrium as a solution. In [5][6], whether spectrum can be fairly and efficiently utilized by modelling the spectrum sharing as a repeated game was investigated. Auction and pricing approaches were also proposed for efficient spectrum allocation [7][8][9]. In [10], auction mechanisms for spectrum sharing among a group of users was studied. A belief-assisted distributive double auction that maximizes both primary and secondary users' revenues was proposed in [11]. To suppress the cheating behaviors in cognitive radio networks, several game theoretic mechanisms have been designed [12][13][14][15]. While these game theoretic approaches have achieved promising results, they cannot be directly used in content-aware multimedia applications since they are designed for data communications but do not explicitly consider the characteristics of the video content and the resulting impact on video quality.

Recently, some works have been proposed for multimedia transmission over cognitive radio networks [16][17][18]. The authors in [16] proposed a queuing-based dynamic channel selection approach by explicitly considering various rate requirements and delay deadlines of heterogeneous multimedia users while those in [17] proposed to jointly optimize application layer quality of service using a partially observable Markov decision process. To compensate the loss due to interference, a distributed multimedia transmission scheme using fountain codes was proposed in [18]. However, all these three approaches are designed under the "Commons" (hierarchical access) spectrum sharing model where the secondary users need to perform sensing and compete with each other to access the spectrum when the primary users are absent. Therefore, they cannot be directly used in the "Exclusive use" spectrum sharing model where the primary users have the rights to sell or trade their spectrum. To address the spectrum allocation problem in the "Exclusive use" spectrum sharing scenario, the authors in [19] proposed a mechanism-based allocation scheme using Vickrey-Clarke-Groves (VCG) auction. In their scheme, the primary user first collects all the private information from the secondary users, and then computes the resource allocated to the secondary users by solving the optimization problem that maximizes the aggregate utility. Moreover, the primary user computes the transfers from every secondary 
user based on the amount of net utility loss it causes other users. Although this approach can achieve promising results, it has several disadvantages: 1) it requires all the secondary users to report all the private information which the secondary users may not be willing to disclose; 2) the primary user needs to solve $N+1$ optimization problems to compute the optimal allocations and transfers, which introduces a lot of computational complexity to the primary user; 3) as shown later in Section V, the scheme is not cheat-proof to the primary user, i.e. the primary user has the incentive to increase the transfers from the secondary users.

In this paper, we specifically consider the unique characteristics of multimedia content and study multimedia streaming over cognitive radio networks under the "Exclusive use" spectrum sharing model, where there is one primary user and $N$ secondary users. In this problem, the objective of the primary user is to maximize his/her revenue by choosing either to self-utilize the spectrum or to sell the spectrum to the secondary users, while the objective of each secondary user is to maximize the payoff by competing with other secondary users to buy the spectrum for streaming.

Our main contributions are summarized as follows.

- We define the utility functions for the primary user and the secondary users by taking into consideration the uniquely scalable and delay-sensitive characteristics of multimedia data and the resulting impact on users' viewing experiences of multimedia content. With such utility functions, the primary user and the secondary users can seamlessly switch among different quality levels to achieve the largest utilities.

- To allocate the spectrum distributively and efficiently, we formulate the spectrum allocation problem as an auction game and propose three spectrum allocation schemes based on auction theory [20][21], which are spectrum allocation using Single object pay-as-bid Ascending Clock Auction (ACA-S), spectrum allocation using Traditional Ascending Clock Auction (ACA-T), and spectrum allocation using Alternative Ascending Clock Auction (ACAA).

- To effectively allocate the spectrum, auction mechanisms should have the convergence property. We prove that all three proposed auction algorithms converges in a finite number of clocks.

- To efficiently utilize the spectrum and yield high revenue to the primary user, auction mechanisms have to allocate the spectrum in an efficient way, e.g. maximizing the social welfare. We prove and demonstrate with simulations that the proposed ACA-T and ACA-A algorithms are able to maximize the social welfare.

- Since the auctions are conducted distributively and users are naturally selfish, enforcing truth-telling is crucial. We prove and demonstrate with simulations that the proposed ACA-S and ACA-A algorithms are cheat-proof.

The rest of this paper is organized as follows. In Section II, we give a detailed description on the system model and the utility function. In Section III, we present the problem formulation and the proposed spectrum allocation schemes. In Section IV, we provide a detailed analysis of the proposed

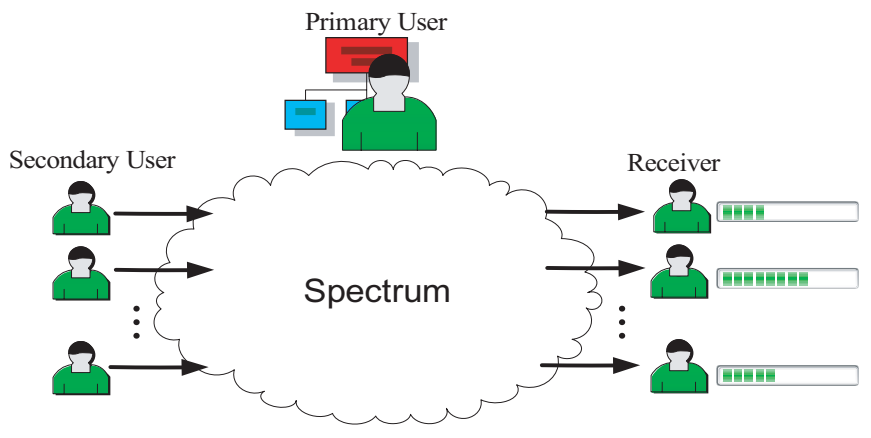

Fig. 1. The system model.

schemes. Finally, we illustrate the simulation results in Section V and draw conclusions in Section VI.

\section{System Model AND Utility Function}

\section{A. System Model}

As shown in Fig. 1, we consider a multimedia cognitive network with one primary user (PU) and $N$ secondary users (SUs), $u_{1}, u_{2}, \ldots, u_{N}$. The PU can choose to utilize the spectrum himself/herself or to sell the available spectrum to SUs who are willing to buy spectrum for streaming multimedia data. In this case, once the PU announces the availability of spectrum, SUs will compete with each other to buy the spectrum. Then, the PU allocates bandwidth to SUs and each SU transmits multimedia streams to the corresponding receiver using the allocated bandwidth. We assume that each SU has a corresponding receiver with a buffer long enough for real-time playback. Now, the problem becomes how and when the PU sells the spectrum as well as how and when the SUs compete with each other to buy the spectrum.

\section{B. Secondary Users' Utility Function}

In general, a SU $u_{i}$ can gain by successfully transmitting the video to the corresponding receiver. On the other hand, $u_{i}$ needs to pay for the used spectrum to transmit video, and the payment is determined by the amount of the used spectrum and its unit price. Therefore, given the bit-rate $r_{i}$, the buffer occupancy at the corresponding receiver $B_{i}$, the allocated bandwidth $W_{i}$, and the unit price $\lambda$, the utility function of $u_{i}$ can be defined as

$$
U_{i}\left(r_{i}, B_{i}, W_{i}, \lambda\right)=\mathcal{F}\left(r_{i}, B_{i}, W_{i}\right)-\mathcal{G}\left(\lambda, W_{i}\right),
$$

where $\mathcal{F}\left(r_{i}, B_{i}, W_{i}\right)$ is the gain, and $\mathcal{G}\left(\lambda, W_{i}\right)$ is the cost. Here, we assume that the source video is compressed using scalable video codec with source rate $\left\{\zeta_{i}^{1}, \ldots, \zeta_{i}^{N_{r}}\right\}$, which means $r_{i} \in\left\{\zeta_{i}^{1}, \ldots, \zeta_{i}^{N_{r}}\right\}$.

Generally speaking, since the cost of $u_{i}$ is larger if the bandwidth $W_{i}$ is larger, the function $\mathcal{G}$ should be a monotonically increasing function of $W_{i}$. In the literature, due to the simplicity and efficiency, linear pricing is widely used [22][23][24]. Moreover, since the primary user does not differentiate among all the bandwidth, it is reasonable to assume that the primary user will sell the bandwidth using a constant unit price, i.e., the cost function of the secondary user is linear, which means

$$
\mathcal{G}\left(\lambda, W_{i}\right)=\lambda W_{i} .
$$


Since two most important factors that reflect the degree of satisfaction of the receiver's video viewing experience are visual quality and delay, we argue that the gain is determined by the visual quality of the transmitted video and the corresponding receiver's buffer occupancy, i.e.

$$
\mathcal{F}\left(r_{i}, B_{i}, W_{i}\right)=\alpha \mathcal{F}_{1}\left(P S N R_{i}\left(r_{i}\right)\right)+\beta \mathcal{F}_{2}\left(r_{i}, B_{i}, W_{i}\right),
$$

where $\mathcal{F}_{1}\left(P S N R_{i}\left(r_{i}\right)\right)$ is the gain due to the effect of visual quality, $\mathcal{F}_{2}\left(r_{i}, B_{i}, W_{i}\right)$ is the gain due to the effect of buffer occupancy, $\alpha$ and $\beta$ are two parameters controlling the balance between $\mathcal{F}_{1}\left(P S N R_{i}\left(r_{i}\right)\right)$ and $\mathcal{F}_{2}\left(r_{i}, B_{i}, W_{i}\right)$.

Since the visual quality difference in the low PSNR region is easier to be distinguished than that in the high PSNR region, we define $\mathcal{F}_{1}\left(P S N R_{i}\left(r_{i}\right)\right)$ as a logarithm function in terms of PSNR by

$$
\mathcal{F}_{1}\left(P S N R_{i}\left(r_{i}\right)\right)=\ln \left(\frac{P S N R_{i}\left(r_{i}\right)}{P S N R_{i}\left(\zeta_{i}^{N_{r}}\right)}\right),
$$

where $\zeta_{i}^{N_{r}}$ is the maximal rate and the $P S N R_{i}\left(\zeta_{i}^{N_{r}}\right)$ in the denominator is for normalization purpose.

Similarly, since the probability of playback delay becomes smaller with more data in the buffer, we define $\mathcal{F}_{2}\left(r_{i}, B_{i}, W_{i}\right)$ as a logarithm function in terms of the buffer occupancy by

$$
\mathcal{F}_{2}\left(r_{i}, B_{i}, W_{i}\right)=\ln \left(\frac{B_{i}+\tau \frac{W_{i}}{r_{i}}+\theta}{B_{i}+\theta}\right),
$$

where $\tau$ is the transmission duration ${ }^{1}, B_{i}+\tau \frac{W_{i}}{r_{i}}$ is the buffer occupancy after transmission, and $\theta$ is a system parameter which excludes the possibility of zero denominator.

Combining (1)-(5), the utility of $u_{i}$ becomes

$$
\begin{aligned}
U_{i}\left(r_{i}, B_{i}, W_{i}, \lambda\right) & =\alpha \ln \left(\frac{P S N R_{i}\left(r_{i}\right)}{P S N R_{i}\left(\zeta_{i}^{N_{r}}\right)}\right) \\
& +\beta \ln \left(\frac{B_{i}+\tau \frac{W_{i}}{r_{i}}+\theta}{B_{i}+\theta}\right)-\lambda W_{i} .
\end{aligned}
$$

\section{Primary User's Utility Function}

Since the PU can choose either to utilize the spectrum himself/herself or to sell the spectrum to $\mathrm{SUs}^{2}$, the utility of PU should be the maximum between the profit $\left(\mathcal{F}_{p}(W)\right)$ that he/she can obtain if he/she choose to self-utilize the spectrum and the payment $(P(W))$ that he/she can obtain if he/she choose to sell the spectrum to SUs, i.e.

$$
U_{p}(W)=\max \left(\mathcal{F}_{p}(W), P(W)\right),
$$

where $W$ is the total bandwidth.

From the above equation, we can see that the PU can at least obtain a profit $\mathcal{F}_{p}(W)$. Therefore, the PU should not sell the spectrum to SUs if $P(W)<\mathcal{F}_{p}(W)$. Let $\lambda^{0}$ stand

\footnotetext{
${ }^{1}$ Note that here we implicitly assume the video streaming model is errorfree. When there are some errors, the transmitter may need to re-transmit the packets. In such a case, the effective transmission duration $\tau$ need to be scaled with a factor which is determined by the expected re-transmission times.

${ }^{2}$ To give more insight into the proposed algorithm, in this paper, we assume that the PU either self-utilize or sell the spectrum as a whole. However, the proposed algorithm can be extended to the case that the PU sell a portion of the spectrum to SUs while reserving the rest.
}

for the lowest unit price (reserve price) at which the PU is willing to sell the spectrum to SUs, then

$$
\lambda^{0}=\frac{\mathcal{F}_{p}(W)}{W} .
$$

Remark: By setting $\lambda^{0}$ as the reserve price, the PU can always get a greater revenue from choosing either to utilize the spectrum himself/herself or to sell the spectrum to the SUs.

\section{Spectrum Auction Games}

In this section, we will discuss how the PU should sell the spectrum. There are two possible approaches, centralized approach and distributed approach. In the centralized approach, the PU knows exactly all the private information of each SU. In such a case, the PU can allocate the spectrum based on some criteria, such as maximizing social welfare or proportional fairness.

However, in general, the SUs can be geographically distributed in many places, it is therefore not feasible for the PU to collect all the private information of each SU. Moreover, since the SUs are selfish, e.g., they tend to overclaim/underclaim what they may need, they will not truly report their private information if cheating can improve their utilities. In this paper, we propose distributed spectrum allocation schemes based on auction theory [20] [21]. An auction is a decentralized mechanism for allocating resources, where there is an auctioneer and several bidders. The auction procedures can be described as follows: the auctioneer announces a price, the bidders report to the auctioneer their demands at that price, and the auctioneer raises the price until the total demand meets the supply. In our spectrum allocation problem, the PU is the auctioneer and the SUs are the bidders.

Specifically, we propose three auction-based distributed spectrum allocation schemes, which are spectrum allocation using Single object pay-as-bid Ascending Clock Auction (ACA-S), spectrum allocation using Traditional Ascending Clock Auction (ACA-T), and spectrum allocation using Alternative Ascending Clock Auction (ACA-A).

\section{A. Spectrum Allocation Using Single Object Pay-as-Bid As- cending Clock Auction (ACA-S)}

The proposed ACA-S scheme is based on the well-known single object pay-as-bid ascending clock auction, where the spectrum is sold as a single object and SUs can only bid 0 or $W$. As shown in Algorithm 1, before the auction, the PU sets up the step size $\delta>0$, clock index $t=0$, initializes $\lambda$ with the reserve price $\lambda^{0}$, and announces $\lambda^{0}$ to all the SUs. Then, each SU computes the maximal utility that he/she can obtain if buying the whole spectrum

$$
U_{i}^{0}=\max _{r_{i}} U_{i}\left(r_{i}, B_{i}, W, \lambda^{0}\right) .
$$

If the utility is positive, then the SU submits his/her optimal bid $W$. Otherwise, the SU submits his/her optimal bid 0 . If less than two SUs bid $W$, the PU concludes the auction and chooses to utilize the spectrum himself/herself.

On the other hand, if more than one SU bid $W$, the PU continues the auction by raising the price $\lambda^{t+1}=\lambda^{t}+\delta$, increasing the clock index $t=t+1$, and announcing $\lambda^{t}$ to all 


\section{Algorithm 1 : Spectrum Allocation Using Single Object Pay-as-Bid Ascending Clock Auction (ACA-S)}

1. Given the available spectrum $W$, step-size $\delta>0$, and clock index $t=0$, the auctioneer initializes the price $\lambda$ with the reserve price $\lambda^{0}$.

2. $u_{i}$ computes $U_{i}^{0}=\max _{r_{i}} U_{i}\left(r_{i}, B_{i}, W, \lambda^{0}\right)$. If $U_{i}^{0}>0, u_{i}$ submits his/her optimal bid $W$. Otherwise, $u_{i}$ submits his/her optimal bid 0 .

3. If less than two SUs bid $W$, the PU concludes the auction and chooses to utilize the spectrum himself/herself. Else, set $\lambda^{t+1}=$ $\lambda^{t}+\delta, t=t+1$, and repeat:

- The PU announces $\lambda^{t}$ to all the SUs.

- Each SU computes $U_{i}^{t}=\max _{r_{i}} U_{i}\left(r_{i}, B_{i}, W, \lambda^{t}\right)$. If $U_{i}^{t}>$ $0, u_{i}$ submits his/her optimal bid $W$. Otherwise, $u_{i}$ submits his/her optimal bid 0 .

- If more than one SU bids $W$, the PU sets $\lambda^{t+1}=\lambda^{t}+\delta$, $t=t+1$, and continues the auction.

- Else, the PU concludes the auction and allocates the spectrum to the SU who bids $W$ at the final clock.

4. Finally, the utility of the SU $u_{i}$ who buys the spectrum is

$U_{i}^{\star}=\alpha \ln \left(\frac{P S N R_{i}\left(r_{i}^{L}\right)}{P S N R_{i}\left(\zeta_{i}^{N_{r}}\right)}\right)+\beta \ln \left(\frac{B_{i}+\tau \frac{W}{r_{i}^{L}}+\theta}{B_{i}+\theta}\right)-\lambda^{L} W$.

where $L$ is the final clock index, and $r_{i}^{L}=$ $\arg \max _{r_{i}} U_{i}\left(r_{i}, B_{i}, W, \lambda^{L}\right)$.

the SUs. Then, each SU submits his/her optimal bid (either 0 or $W$ ) by checking the sign of the utility

$$
U_{i}^{t}=\max _{r_{i}} U_{i}\left(r_{i}, B_{i}, W, \lambda^{t}\right) .
$$

The auction is repeated until there is only one SU bidding $W$. And the spectrum is allocated to the SU who bids $W$ at the final clock.

\section{B. Spectrum Allocation Using Traditional Ascending Clock Auction (ACA-T)}

From the previous subsection, we can see that the spectrum is sold as a single object in the ACA-S scheme, which may lead to inefficient spectrum allocation since the SUs may need only part of rather than the whole spectrum. To address this problem, the ACA-T scheme using traditional ascending clock auction is proposed, where each SU is allowed to bid any value between 0 and $W$ at every clock.

As shown in Algorithm 2, when the PU announces the reserve price $\lambda^{0}$, each SU submits his/her optimal bid $W_{i}^{0}$ by computing

$$
\left(W_{i}^{0}, r_{i}^{0}\right)=\arg \max _{\left(W_{i}, r_{i}\right)} U_{i}\left(r_{i}, B_{i}, W_{i}, \lambda^{0}\right) .
$$

Then, the PU sums up all the bids $W_{\text {total }}^{0}=\sum_{i} W_{i}^{0}$ and compares $W_{\text {total }}^{0}$ with $W$. If $W_{\text {total }}^{0} \leq W$, the PU concludes the auction and chooses to utilize the spectrum himself/herself. Otherwise, the PU sets $\lambda^{t+1}=\lambda^{t}+\delta, t=t+1$, and announces $\lambda^{t}$ to all the SUs. Then, each SU submits his/her optimal bid $W_{i}^{t}$ to the PU by calculating

$$
\left(W_{i}^{t}, r_{i}^{t}\right)=\arg \max _{\left(W_{i}, r_{i}\right)} U_{i}\left(r_{i}, B_{i}, W_{i}, \lambda^{t}\right) .
$$

After collecting all the bids, the PU compares the total bid $W_{\text {total }}^{t}$ with the available bandwidth $W$. If $W_{\text {total }}^{t}>W$, the

\section{Algorithm 2 : Spectrum Allocation Using Traditional Ascending Clock Auction (ACA-T)}

1. Given the available spectrum $W$, step-size $\delta>0$, and clock index $t=0$, the PU initializes the price $\lambda$ with the reserve price $\lambda^{0}$.

2. $u_{i}$ computes

$$
\left(W_{i}^{0}, r_{i}^{0}\right)=\arg \max _{\left(W_{i}, r_{i}\right)} U_{i}\left(r_{i}, B_{i}, W_{i}, \lambda^{0}\right)
$$

and submits his/her optimal bid $W_{i}^{0}$

3 . The PU sums up all the bids $W_{\text {total }}^{0}=\sum_{i} W_{i}^{0}$ and compares $W_{\text {total }}^{0}$ with $W$.

- If $W_{\text {total }}^{0} \leq W$, the PU concludes the auction and chooses to utilize the spectrum himself/herself.

- Else, set $\lambda^{t+1}=\lambda^{t}+\delta, t=t+1$, and repeat:

- The PU announces $\lambda^{t}$ to all the SUs.

- Each SU computes

$$
\left(W_{i}^{t}, r_{i}^{t}\right)=\arg \max _{\left(W_{i}, r_{i}\right)} U_{i}\left(r_{i}, B_{i}, W_{i}, \lambda^{t}\right)
$$

and submits his/her optimal bid $W_{i}^{t}$.

- The PU sums up all the bids $W_{\text {total }}^{t}=\sum_{i} W_{i}^{t}$ and compares $W_{\text {total }}^{t}$ with $W$ :

* If $W_{\text {total }}^{t}>W$, set $\lambda^{t+1}=\lambda^{t}+\delta, t=t+1$, and continue the auction.

* Else, conclude the auction, set $L=t$, and allocate $W_{i}^{\star}=W_{i}^{L}+\frac{W_{i}^{L-1}-W_{i}^{L}}{\sum_{i} W_{i}^{L-1}-\sum_{i} W_{i}^{L}}\left[W-\sum_{i} W_{i}^{L}\right]$ to $u_{i}$.

4. Finally, the utility of $u_{i}$ is

$$
U_{i}^{\star}=\alpha \ln \left(\frac{P S N R_{i}\left(r_{i}^{L}\right)}{\operatorname{PSNR} R_{i}\left(\zeta_{i}^{N_{r}}\right)}\right)+\beta \ln \left(\frac{B_{i}+\tau \frac{W_{i}^{\star}}{r_{i}^{L}}+\theta}{B_{i}+\theta}\right)-\lambda^{L} W_{i}^{\star} .
$$

auction is not concluded. The PU continues the auction until $W_{\text {total }}^{t} \leq W$. Let the final clock index be $L$. As $\lambda$ increases discretely, we may have $W_{\text {total }}^{t}<W$ and do not fully utilize the bandwidth. To make sure that $W_{\text {total }}^{t}=W$, we modify $W_{i}^{L}$ by introducing proportional rationing [21]. Then, the final allocated bandwidth of $u_{i}$ is given by

$$
W_{i}^{\star}=W_{i}^{L}+\frac{W_{i}^{L-1}-W_{i}^{L}}{\sum_{i} W_{i}^{L-1}-\sum_{i} W_{i}^{L}}\left[W-\sum_{i} W_{i}^{L}\right],
$$

with $\sum_{i} W_{i}^{\star}=W$.

Consequently, the utility of $u_{i}$ is

$U_{i}^{\star}=\alpha \ln \left(\frac{P S N R_{i}\left(r_{i}^{L}\right)}{P S N R_{i}\left(\zeta_{i}^{N_{r}}\right)}\right)+\beta \ln \left(\frac{B_{i}+\tau \frac{W_{i}^{\star}}{r_{i}^{L}}+\theta}{B_{i}+\theta}\right)-\lambda^{L} W_{i}^{\star}$

\section{Spectrum Allocation Using Alternative Ascending Clock Auction (ACA-A)}

Note that the ACA-T algorithm shown in the previous subsection is equivalent to the distributed dual-based optimization approach for Network Utility Maximization (NUM) problem [25] [26], which means that ACA-T can achieve efficient spectrum allocation. However, as we will prove in the next section and verify in the simulation results, ACA-T is not cheat-proof.

To overcome the drawback of the ACA-T scheme, the ACA-A scheme using alternative ascending clock auction 


\section{Algorithm 3 : Spectrum Allocation Using Alternative Ascending Clock Auction (ACA-A)}

1. Given the available spectrum $W$, step-size $\delta>0$, and clock index $t=0$, the PU initializes the price $\lambda$ with the reserve price $\lambda^{0}$.

2. $u_{i}$ computes

$$
\left(W_{i}^{0}, r_{i}^{0}\right)=\arg \max _{\left(W_{i}, r_{i}\right)} U_{i}\left(r_{i}, B_{i}, W_{i}, \lambda^{0}\right)
$$

and submits his/her optimal bid $W_{i}^{0}$.

3. The PU sums up all the bids $W_{\text {total }}^{0}=\sum_{i} W_{i}^{0}$ and compares $W_{\text {total }}^{0}$ with $W$.

- If $W_{\text {total }}^{0} \leq W$, the PU concludes the auction and chooses to utilize the spectrum himself/herself.

- Else, set $\lambda^{t+1}=\lambda^{t}+\delta, t=t+1$, and repeat:

- The PU announces $\lambda^{t}$ to all the SUs.

- Each SU computes

$$
\left(W_{i}^{t}, r_{i}^{t}\right)=\arg \max _{\left(W_{i}, r_{i}\right)} U_{i}\left(r_{i}, B_{i}, W_{i}, \lambda^{t}\right)
$$

and submits his/her optimal bid $W_{i}^{t}$.

- The PU sums up all the bids $W_{\text {total }}^{t}=\sum_{i} W_{i}^{t}$ and compares $W_{\text {total }}^{t}$ with $W$ :

* If $W_{\text {total }}^{t}>W$, compute $C_{i}^{t}=\max \left(0, W-\sum_{j \neq i} W_{j}^{t}\right)$, set $\lambda^{t+1}=\lambda^{t}+\delta, t=t+1$, and continue the auction. * Else, conclude the auction, set $L=t$, compute $C_{i}^{L}=$ $W_{i}^{L}+\frac{W_{i}^{L-1}-W_{i}^{L}}{\sum_{i} W_{i}^{L-1}-\sum_{i} W_{i}^{L}}\left[W-\sum_{i} W_{i}^{L}\right]$, and allocate $W_{i}^{\star}=C_{i}^{L}$ to $u_{i}$

4. Finally, the payment of $u_{i}$ is

$$
P_{i}^{\star}=C_{i}^{0} \lambda^{0}+\sum_{t=1}^{L} \lambda^{t}\left(C_{i}^{t}-C_{i}^{t-1}\right)
$$

and the utility of $u_{i}$ is

$$
U_{i}^{\star}=\alpha \ln \left(\frac{P S N R_{i}\left(r_{i}^{L}\right)}{P S N R_{i}\left(\zeta_{i}^{N_{r}}\right)}\right)+\beta \ln \left(\frac{B_{i}+\tau \frac{W_{i}^{\star}}{r_{i}^{L}}+\theta}{B_{i}+\theta}\right)-P_{i}^{\star} .
$$

is proposed and described in details in Algorithm 3. The procedures of ACA-A are the same as ACA-T except that at every clock $t$ in ACA-A, the PU computes the cumulative clinch, which is the amount of bandwidth that the user is guaranteed to win at clock $t$, for each SU using

$$
C_{i}^{t}=\max \left(0, W-\sum_{j \neq i} W_{j}^{t}\right) .
$$

Similar to (13), to make sure that $W_{\text {total }}^{t}=W$ at final clock $L$, the final cumulative clinch of $u_{i}$ is given by

$$
C_{i}^{L}=W_{i}^{L}+\frac{W_{i}^{L-1}-W_{i}^{L}}{\sum_{i} W_{i}^{L-1}-\sum_{i} W_{i}^{L}}\left[W-\sum_{i} W_{i}^{L}\right],
$$

with $\sum_{i} C_{i}^{L}=W$.

Finally, the rate allocated to $u_{i}$ is $W_{i}^{\star}=C_{i}^{L}$ and the utility of $u_{i}$ is computed by

$$
U_{i}^{\star}=\alpha \ln \left(\frac{P S N R_{i}\left(r_{i}^{L}\right)}{P S N R_{i}\left(\zeta_{i}^{N_{r}}\right)}\right)+\beta \ln \left(\frac{B_{i}+\tau \frac{W_{i}^{\star}}{r_{i}^{L}}+\theta}{B_{i}+\theta}\right)-P_{i}^{\star},
$$

where $P_{i}^{\star}=C_{i}^{0} \lambda^{0}+\sum_{t=1}^{L} \lambda^{t}\left(C_{i}^{t}-C_{i}^{t-1}\right)$ is the payment from $u_{i}$.
Note that with the cumulative clinch, we will show in the following section that ACA-A is cheat-proof.

\section{Analysis of the Spectrum Auction Games}

According to (6), we can see that for any fixed $r_{i}$, the utility function $U_{i}\left(r_{i}, B_{i}, W_{i}, \lambda\right)$ is a concave function in terms of $W_{i}$. By taking the derivative of $U_{i}$ over $W_{i}$, we have

$$
\frac{\partial U_{i}}{\partial W_{i}}=\frac{\beta \frac{\tau}{r_{i}}}{B_{i}+\frac{\tau}{r_{i}} W_{i}+\theta}-\lambda .
$$

Therefore, for any fixed $r_{i}, U_{i}\left(r_{i}, B_{i}, W_{i}, \lambda\right)$ achieves the maximal value at

$$
W_{i}^{\star}\left(r_{i}, \lambda\right)=\min \left(W, \max \left(0, \frac{\beta}{\lambda}-\frac{B_{i}+\theta}{\tau} r_{i}\right)\right) .
$$

By substituting (19) back to the utility function, we can find the optimal $r_{i}^{\star}$ that maximizes the utility function

$$
r_{i}^{\star}(\lambda)=\arg \max _{r_{i}} f\left(r_{i}, \lambda\right)
$$

where $f\left(r_{i}, \lambda\right)$ is defined in (21).

Then, the optimal $W_{i}^{\star}$ that achieves the maximal utility becomes

$$
W_{i}^{\star}(\lambda)=\min \left(W, \max \left(0, \frac{\beta}{\lambda}-\frac{B_{i}+\theta}{\tau} r_{i}^{\star}(\lambda)\right)\right),
$$

where $r_{i}^{\star}(\lambda)$ is defined in (20).

In the following subsections, we will discuss three important properties of the three proposed algorithms (ACA-S, ACA$\mathrm{T}$, and $\mathrm{ACA}-\mathrm{A})$ : convergence, cheat-proof, and maximizing social welfare.

\section{A. Convergence}

In this subsection, we prove that all three algorithms (ACA$\mathrm{S}, \mathrm{ACA}-\mathrm{T}$, and ACA-A) have the convergence property.

Theorem 1: The ACA-S algorithm will conclude in a finite number of clocks.

Proof: According to (10), we know

$$
\begin{aligned}
& U_{i}^{t}=\max _{r_{i}} U_{i}\left(r_{i}, B_{i}, W, \lambda^{t}\right) \\
& =\max _{r_{i}}\left[\alpha \ln \left(\frac{P S N R_{i}\left(r_{i}\right)}{P S N R_{i}\left(\zeta_{i}^{N_{r}}\right)}\right)+\beta \ln \left(\frac{B_{i}+\tau \frac{W}{r_{i}}+\theta}{B_{i}+\theta}\right)-\lambda^{t} W\right] .
\end{aligned}
$$

Therefore, we have

$$
U_{i}^{t+1}-U_{i}^{t}=-\delta W<0 .
$$

According to Algorithm 1, we know that $W_{i}^{t}=W$ if $U_{i}^{t}>$ 0 and $W_{i}^{t}=0$ if $U_{i}^{t} \leq 0$. Since $U_{i}^{t+1}<U_{i}^{t}$, with sufficiently large $t, W_{i}^{t+1}=0 \leq W_{i}^{t}$. Therefore, there exists a finite number $L$ such that $\sum_{i=1}^{N} W_{i}^{L}=W$, which means that the auction concludes at clock $L$.

Lemma 1: In ACA-T and ACA-A, the optimal $r_{i}^{t}$ is a nondecreasing function in terms of the clock index $t$, i.e. $r_{i}^{t+1} \geq$ $r_{i}^{t}, \forall t$.

Proof: To prove the above Lemma, let us first define $g\left(r_{i}, \lambda, \delta\right)=f\left(r_{i}, \lambda+\delta\right)-f\left(r_{i}, \lambda\right)$ with $\delta>0$, which can be computed as shown in (23). The derivative of $g\left(r_{i}, \lambda, \delta\right)$ over $r_{i}$ is shown in (24). 


$$
f\left(r_{i}, \lambda\right)= \begin{cases}\alpha \ln \left(\frac{P S N R_{i}\left(r_{i}\right)}{P S N R_{i}\left(\zeta_{i}^{N}\right)}\right)+\beta \ln \left(B_{i}+\tau \frac{W}{r_{i}}+\theta\right)-\lambda W-\beta \ln \left(B_{i}+\theta\right), & \text { if } \frac{\beta}{\lambda}-\frac{B_{i}+\theta}{\tau} r_{i}>W \\ \alpha \ln \left(\frac{P S N R_{i}\left(r_{i}\right)}{P S N R_{i}\left(\zeta_{i}^{N r}\right)}\right)+\beta \ln \left(\frac{\beta \tau}{\lambda r_{i}}\right)-\beta+\lambda \frac{B_{i}+\theta}{\tau} r_{i}-\beta \ln \left(B_{i}+\theta\right), & \text { if } 0 \leq \frac{\beta}{\lambda}-\frac{B_{i}+\theta}{\tau} r_{i} \leq W \\ \alpha \ln \left(\frac{P S N R_{i}\left(r_{i}\right)}{P S N R_{i}\left(\zeta_{i}^{N r}\right)}\right), & \text { if } \frac{\beta}{\lambda}-\frac{B_{i}+\theta}{\tau} r_{i}<0\end{cases}
$$

$$
g\left(r_{i}, \lambda, \delta\right)=\left\{\begin{array}{l}
-\delta W, \\
\beta \ln \left(\frac{\beta \tau}{(\lambda+\delta) r_{i}}\right)-\beta+(\lambda+\delta) \frac{B_{i}+\theta}{\tau} r_{i} \\
\quad-\beta \ln \left(B_{i}+\tau \frac{W}{r_{i}}+\theta\right)+\lambda W \\
\beta \ln \left(\frac{\lambda}{\lambda+\delta}\right)+\delta \frac{B_{i}+\theta}{\tau} r_{i} \\
\beta \ln \left(B_{i}+\theta\right)-\beta \ln \left(\frac{\beta \tau}{\lambda r_{i}}\right)+\beta-\lambda \frac{B_{i}+\theta}{\tau} r_{i} \\
0,
\end{array}\right.
$$

if $\frac{\beta}{\lambda}-\frac{B_{i}+\theta}{\tau} r_{i}>W, \frac{\beta}{\lambda+\delta}-\frac{B_{i}+\theta}{\tau} r_{i}>W$;

if $\frac{\beta}{\lambda}-\frac{B_{i}+\theta}{\tau} r_{i}>W, 0 \leq \frac{\beta}{\lambda+\delta}-\frac{B_{i}+\theta}{\tau} r_{i} \leq W$;

if $0 \leq \frac{\beta}{\lambda}-\frac{B_{i}+\theta}{\tau} r_{i} \leq W, 0 \leq \frac{\beta}{\lambda+\delta}-\frac{B_{i}+\theta}{\tau} r_{i} \leq W$;

if $0 \leq \frac{\beta}{\lambda}-\frac{B_{i}+\theta}{\tau} r_{i} \leq W, \frac{\beta}{\lambda+\delta}-\frac{B_{i}+\theta}{\tau} r_{i}<0$;

if $\frac{\beta}{\lambda}-\frac{B_{i}+\theta}{\tau} r_{i}<0, \frac{\beta}{\lambda+\delta}-\frac{B_{i}+\theta}{\tau} r_{i}<0$.

$$
\frac{\partial g\left(r_{i}, \lambda, \delta\right)}{\partial r_{i}}=\left\{\begin{array}{l}
0, \\
-\frac{\beta}{r_{i}}+(\lambda+\delta) \frac{B_{i}+\theta}{\tau}+\frac{\beta W}{r_{i}\left(\frac{B_{i}+\theta}{\tau} r_{i}+W\right)} \geq 0 \\
\delta \frac{B_{i}+\theta}{\tau}>0 \\
\frac{\lambda}{r_{i}}\left(\frac{\beta}{\lambda}-\frac{B_{i}+\theta}{\tau} r_{i}\right) \geq 0 \\
0,
\end{array}\right.
$$

From (24), we can see that $\frac{\partial g\left(r_{i}, \lambda, \delta\right)}{\partial r_{i}} \geq 0$, which means $g\left(r_{i}, \lambda, \delta\right)$ is a non-decreasing function in terms of $r_{i}$. Therefore, $\forall r_{i} \leq r_{i}^{t}$, we have

$$
g\left(r_{i}^{t}, \lambda^{t}, \delta\right) \geq g\left(r_{i}, \lambda^{t}, \delta\right),
$$

which means

$$
f\left(r_{i}^{t}, \lambda^{t+1}\right)-f\left(r_{i}, \lambda^{t+1}\right) \geq f\left(r_{i}^{t}, \lambda^{t}\right)-f\left(r_{i}, \lambda^{t}\right) .
$$

According to (20), we have

$$
\begin{aligned}
r_{i}^{t} & =\arg \max _{r_{i}} f\left(r_{i}, \lambda^{t}\right), \\
r_{i}^{t+1} & =\arg \max _{r_{i}} f\left(r_{i}, \lambda^{t+1}\right),
\end{aligned}
$$

which means

$$
\begin{array}{r}
f\left(r_{i}^{t}, \lambda^{t}\right) \geq f\left(r_{i}, \lambda^{t}\right), \\
f\left(r_{i}^{t+1}, \lambda^{t+1}\right) \geq f\left(r_{i}^{t}, \lambda^{t+1}\right) .
\end{array}
$$

According to (26) and (28), we have

$$
f\left(r_{i}^{t+1}, \lambda^{t+1}\right) \geq f\left(r_{i}^{t}, \lambda^{t+1}\right) \geq f\left(r_{i}, \lambda^{t+1}\right), \forall r_{i} \leq r_{i}^{t} .
$$

Therefore, we have

$$
r_{i}^{t+1} \geq r_{i}^{t}, \forall t
$$

Lemma 2: In ACA-T and ACA-A, the optimal bid $W_{i}^{t}$ is a non-increasing function in terms of the clock index $t$, i.e. $W_{i}^{t+1} \leq W_{i}^{t}$, with equality holds when $W_{i}^{t+1}=W_{i}^{t}=0$ or $W_{i}^{t+1}=W_{i}^{t}=W, \forall t$.

Proof: According to (22),

$$
W_{i}^{t+1}=\min \left(W, \max \left(0, \frac{\beta}{\lambda^{t+1}}-\frac{B_{i}+\theta}{\tau} r_{i}^{t+1}\right)\right) .
$$

if $\frac{\beta}{\lambda}-\frac{B_{i}+\theta}{\tau} r_{i}>W, \frac{\beta}{\lambda+\delta}-\frac{B_{i}+\theta}{\tau} r_{i}>W$;

if $\frac{\beta}{\lambda}-\frac{B_{i}+\theta}{\tau} r_{i}>W, 0 \leq \frac{\beta}{\lambda+\delta}-\frac{B_{i}+\theta}{\tau} r_{i} \leq W$;

if $0 \leq \frac{\beta}{\lambda}-\frac{B_{i}+\theta}{\tau} r_{i} \leq W, 0 \leq \frac{\beta}{\lambda+\delta}-\frac{B_{i}+\theta}{\tau} r_{i} \leq W$;

if $0 \leq \frac{\beta}{\lambda}-\frac{B_{i}+\theta}{\tau} r_{i} \leq W, \frac{\beta}{\lambda+\delta}-\frac{B_{i}+\theta}{\tau} r_{i}<0$;

if $\frac{\beta}{\lambda}-\frac{B_{i}+\theta}{\tau} r_{i}<0, \frac{\beta}{\lambda+\delta}-\frac{B_{i}+\theta}{\tau} r_{i}<0$.
Since $\lambda^{t+1}>\lambda^{t}$ and $r_{i}^{t+1} \geq r_{i}^{t}$ (according to Lemma 1), we have

$$
W_{i}^{t+1} \leq W_{i}^{t}
$$

with equality holds when $W_{i}^{t+1}=W_{i}^{t}=0$ or $W_{i}^{t+1}=W_{i}^{t}=$ $W$.

Theorem 2: The ACA-T and ACA-A algorithms will conclude in a finite number of clocks.

Proof: According to Lemma 2, $W_{i}^{t+1} \leq W_{i}^{t}$, with equality holds when $W_{i}^{t+1}=W_{i}^{t}=0$ or $W_{i}^{t+1}=W_{i}^{t}=W$, $\forall t$. Since $\lambda$ increases with a fixed step size $\delta>0$, with sufficiently large $t, W_{i}^{t+1}<W_{i}^{t}<W, \forall i$. Therefore, there exists a finite number $L$ such that $\sum_{i=1}^{N} W_{i}^{L} \leq W$, which means that the auction concludes at clock $L$.

\section{B. Cheat-Proof Property}

In this subsection, we prove that ACA-S and ACA-A algorithms are cheat-proof while ACA-T algorithm is not.

Theorem 3: ACA-S algorithm is cheat-proof.

Proof: Since single object pay-as-bid ascending clock auction is equivalent to second price sealed-bid auction which is cheat-proof [20], ACA-S is also cheat-proof.

Theorem 4: In ACA-A algorithm, reporting true optimal demand at every clock is a mutually best response for every user, i.e. ACA-A algorithm is cheat-proof.

Proof: Given that all other users report their true optimal demands at every clock, let us assume that the auction will conclude at clock $L_{1}$ if $u_{i}$ also reports his/her true optimal demands at every clock and the utility of $u_{i}$ is $U_{i}\left(L_{1}\right)$. Let us assume that the auction will conclude at clock $L_{2}$ if $u_{i}$ does not report his/her true optimal demands at every clock and the 
utility of $u_{i}$ is $U_{i}\left(L_{2}\right)$. According to (17), we have

$$
\begin{aligned}
U_{i}\left(L_{j}\right) & =\alpha \ln \left(\frac{P S N R_{i}\left(r_{i}^{L_{j}}\right)}{P S N R_{i}\left(\zeta_{i}^{N_{r}}\right)}\right)+\beta \ln \left(\frac{B_{i}+\tau \frac{W_{i}^{L_{j}}}{r_{i}^{L_{j}}}+\theta}{B_{i}+\theta}\right) \\
& -C_{i}^{0} \lambda^{0}-\sum_{t=1}^{L_{j}} \lambda^{t}\left(C_{i}^{t}-C_{i}^{t-1}\right), j \in\{1,2\} .
\end{aligned}
$$

When $\delta$ is sufficiently small, we have

$$
C_{i}^{L_{j}}=W_{i}^{L_{j}}=W-\sum_{k=1, k \neq i}^{N} W_{k}^{L_{j}}, j \in\{1,2\} .
$$

- If $L_{2}<L_{1}$, according to Lemma 2 and (34), we have $W_{i}^{L_{2}} \leq W_{i}^{L_{1}}$. Then,

$$
\begin{aligned}
& U_{i}\left(L_{1}\right)-U_{i}\left(L_{2}\right) \\
& =\alpha \ln \left(\frac{P S N R_{i}\left(r_{i}^{L_{1}}\right)}{P S N R_{i}\left(r_{i}^{L_{2}}\right)}\right)+\beta \ln \left(\frac{B_{i}+\tau \frac{W_{i}^{L_{1}}}{r_{i}^{L_{1}}}+\theta}{B_{i}+\tau \frac{W_{i}^{L_{2}}}{r_{i}^{L_{2}}}+\theta}\right) \\
& -\sum_{t=L_{2}+1}^{L_{1}} \lambda^{t}\left(C_{i}^{t}-C_{i}^{t-1}\right), \\
& >\alpha \ln \left(P S N R_{i}\left(r_{i}^{L_{1}}\right)\right)+\beta \ln \left(B_{i}+\tau \frac{W_{i}^{L_{1}}}{r_{i}^{L_{1}}}+\theta\right)-\lambda^{L_{1}} W_{i}^{L_{1}} \\
& -\alpha \ln \left(P S N R_{i}\left(r_{i}^{L_{2}}\right)\right)-\beta \ln \left(B_{i}+\tau \frac{W_{i}^{L_{2}}}{r_{i}^{L_{2}}}+\theta\right)+\lambda^{L_{1}} W_{i}^{L_{2}}, \\
& =U_{i}\left(r_{i}^{L_{1}}, B_{i}, W_{i}^{L_{1}}, \lambda^{L_{1}}\right)-U_{i}\left(r_{i}^{L_{2}}, B_{i}, W_{i}^{L_{2}}, \lambda^{L_{1}}\right), \\
& \geq 0,
\end{aligned}
$$

where the last inequality comes from (12) that $\left(W_{i}^{L_{1}}, r_{i}^{L_{1}}\right)=\arg \max _{\left(W_{i}, r_{i}\right)} U_{i}\left(r_{i}, B_{i}, W_{i}, \lambda^{L_{1}}\right)$.

- If $L_{2} \geq L_{1}$, according to Lemma 2 and (34), we have $W_{i}^{L_{2}} \geq W_{i}^{L_{1}}$. Then,

$$
\begin{aligned}
& U_{i}\left(L_{1}\right)-U_{i}\left(L_{2}\right) \\
& =\alpha \ln \left(\frac{P S N R_{i}\left(r_{i}^{L_{1}}\right)}{P S N R_{i}\left(r_{i}^{L_{2}}\right)}\right)+\beta \ln \left(\frac{B_{i}+\tau \frac{W_{i}^{L_{1}}}{r_{i}^{L_{1}}}+\theta}{B_{i}+\tau \frac{W_{i}^{L_{2}}}{\tilde{r}_{i}^{L_{2}}}+\theta}\right) \\
& +\sum_{t=L_{1}+1}^{L_{2}} \lambda^{t}\left(C_{i}^{t}-C_{i}^{t-1}\right), \\
& >\alpha \ln \left(P S N R_{i}\left(r_{i}^{L_{1}}\right)\right)+\beta \ln \left(B_{i}+\tau \frac{W_{i}^{L_{1}}}{r_{i}^{L_{1}}}+\theta\right)-\lambda^{L_{1}} W_{i}^{L_{1}} \\
& -\alpha \ln \left(P S N R_{i}\left(r_{i}^{L_{2}}\right)\right)-\beta \ln \left(B_{i}+\tau \frac{W_{i}^{L_{2}}}{r_{i}^{L_{2}}}+\theta\right)+\lambda^{L_{1}} W_{i}^{L_{2}}, \\
& =U_{i}\left(r_{i}^{L_{1}}, B_{i}, W_{i}^{L_{1}}, \lambda^{L_{1}}\right)-U_{i}\left(r_{i}^{L_{2}}, B_{i}, W_{i}^{L_{2}}, \lambda^{L_{1}}\right), \\
& \geq 0,
\end{aligned}
$$

where the last inequality comes from (12) that $\left(W_{i}^{L_{1}}, r_{i}^{L_{1}}\right)=\arg \max _{\left(W_{i}, r_{i}\right)} U_{i}\left(r_{i}, B_{i}, W_{i}, \lambda^{L_{1}}\right)$.

In all, according to (35) and (36), we can show that $U_{i}\left(L_{1}\right)>U_{i}\left(L_{2}\right)$. Therefore, given that all other users report their true optimal demands at every clock, the best strategy of $u_{i}$ is to report his/her true optimal demands at every clock. Since all users are non-collaborative, reporting true optimal demand at every clock is a mutually best response for every user. There is no incentive for the users to cheat since any cheating may lead to a loss in utility. Therefore, ACA-A algorithm is cheat-proof.

Theorem 5: ACA-T algorithm is not cheat-proof.

Proof: Given that all other users report their true optimal demands at every clock, let us assume that the auction will conclude with a price $\lambda^{L_{1}}$ and spectrum allocation $W_{i}^{L_{1}}$ if $u_{i}$ also report his/her true optimal demands at every clock and the utility of $u_{i}$ is $U_{i}\left(L_{1}\right)$. Let us assume that the auction will conclude with a price $\lambda^{L_{2}}$ and spectrum allocation $W_{i}^{L_{2}}$ if $u_{i}$ does not report his/her true optimal demands at every clock and the utility of $u_{i}$ is $U_{i}\left(L_{2}\right)$. According to Algorithm 2, for any fixed $r_{i}$, we have

$$
\begin{aligned}
U_{i}\left(L_{j}\right)=\alpha & \ln \left(\frac{P S N R_{i}\left(r_{i}\right)}{P S N R_{i}\left(\zeta_{i}^{N_{r}}\right)}\right)+\beta \ln \left(\frac{B_{i}+\tau \frac{W_{i}^{L_{j}}}{r_{i}}+\theta}{B_{i}+\theta}\right) \\
& -\lambda^{L_{j}} W_{i}^{L_{j}}, j \in\{1,2\} .
\end{aligned}
$$

Therefore, we have

$$
\begin{aligned}
U_{i}\left(L_{1}\right)-U_{i}\left(L_{2}\right)= & \beta \ln \left(\frac{B_{i}+\tau \frac{W_{i}^{L_{1}}}{r_{i}}+\theta}{B_{i}+\tau \frac{W_{i}^{L_{2}}}{r_{i}}+\theta}\right) \\
& -\lambda^{L_{1}} W_{i}^{L_{1}}+\lambda^{L_{2}} W_{i}^{L_{2}} .
\end{aligned}
$$

From (38), we can see that we can not guarantee $U_{i}\left(L_{1}\right)>$ $U_{i}\left(L_{2}\right)$ since if $\lambda^{L_{2}} W_{i}^{L_{2}}<-\beta \ln \left(\frac{B_{i}+\tau \frac{W_{i}^{L_{1}}}{r_{i}}+\theta}{B_{i}+\tau \frac{W_{i}^{L_{2}}}{r_{i}}+\theta}\right)+$ $\lambda^{L_{1}} W_{i}^{L_{1}}$, then $U_{i}\left(L_{1}\right)<U_{i}\left(L_{2}\right)$. Therefore, ACA-T is not cheat-proof. All SUs have the incentive to deviate since it may lead to a greater utility.

\section{Maximizing Social Welfare}

In this subsection, we prove that ACA-T and ACA-A maximize the social welfare, which is the sum of the PU's and SUs' utilities, while ACA-S achieves a smaller, if not equal, social welfare.

Theorem 6: When $\delta$ is sufficiently small, ACA-T and ACAA will converge to $\left(W_{1}^{\star}, r_{1}^{\star}, \ldots, W_{N}^{\star}, r_{N}^{\star}\right)$, which maximizes the social welfare, i.e. $\left(W_{1}^{\star}, r_{1}^{\star}, \ldots, W_{N}^{\star}, r_{N}^{\star}\right)$ is the solution to the following optimization problem

$$
\begin{aligned}
& \max _{\left(W_{i}, r_{i} \forall i\right)} \sum_{i=1}^{N}\left[\alpha \ln \left(\frac{P S N R_{i}\left(r_{i}\right)}{P S N R_{i}\left(\zeta_{i}^{N_{r}}\right)}\right)+\beta \ln \left(\frac{B_{i}+\tau \frac{W_{i}}{r_{i}}+\theta}{B_{i}+\theta}\right)\right], \\
& \text { s.t. } \quad 0 \leq W_{i} \leq W, \forall i=1, \ldots, N \\
& \quad \sum_{i=1}^{N} W_{i}=W .
\end{aligned}
$$

Proof: According to Theorem 2, we know that the auction in ACA-T and ACA-A will conclude in a finite number of clocks, which means that the auction will converge to a solution $\left(W_{1}^{\star}, r_{1}^{\star}, \ldots, W_{N}^{\star}, r_{N}^{\star}\right)$. From (22), we can see that $W_{i}^{\star}(\lambda)=\min \left(W, \max \left(0, \frac{\beta}{\lambda}-\frac{B_{i}+\theta}{\tau} r_{i}^{\star}(\lambda)\right)\right)$ where $r_{i}^{\star}(\lambda)$ 


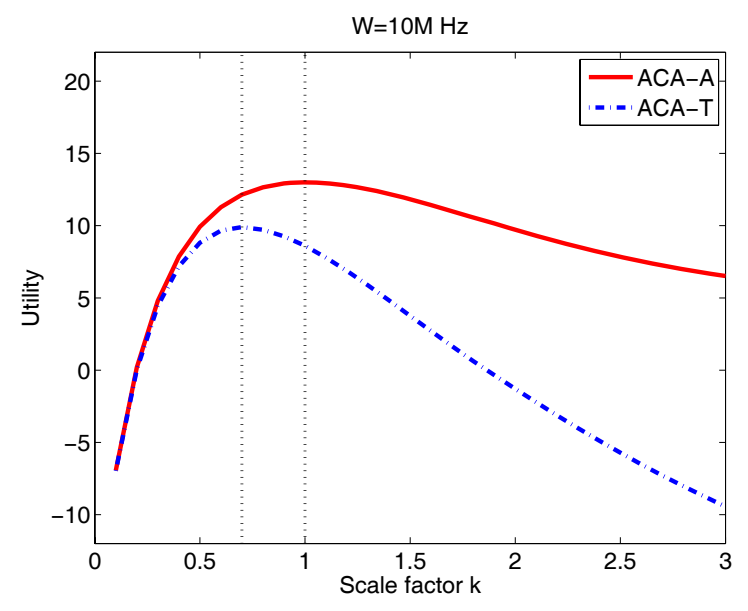

Fig. 2. The cheat-proof performance of ACA-A and ACA-T algorithms.

is defined in (20) and $\lambda$ is the solution to the following equation

$$
\sum_{i=1}^{N} \min \left(W, \max \left(0, \frac{\beta}{\lambda}-\frac{B_{i}+\theta}{\tau} r_{i}^{\star}(\lambda)\right)\right)=W .
$$

Since the optimization problem in (39) is convex in terms of $W_{i}$ for any fixed $r_{i}$, we first find the optimal $W_{i}$ as a function of $r_{i}$ by solving the Karush- Kuhn-Tucker (KKT) conditions [27]. We first write the Lagrangian of problem (39) as

$$
\begin{aligned}
& L\left(W_{i}, r_{i}, \lambda, \kappa_{i}, \nu_{i}\right) \\
& =-\sum_{i=1}^{N}\left[\alpha \ln \left(\frac{P S N R_{i}\left(r_{i}\right)}{P S N R_{i}\left(\zeta_{i}^{N_{r}}\right)}\right)+\beta \ln \left(\frac{B_{i}+\tau \frac{W_{i}}{r_{i}}+\theta}{B_{i}+\theta}\right)\right] \\
& +\lambda\left(\sum_{i=1}^{N} W_{i}-W\right)+\sum_{i=1}^{N} \kappa_{i}\left(W_{i}-W\right)-\sum_{i=1}^{N} \nu_{i} W_{i} .
\end{aligned}
$$

Then, the KKT conditions are:

$$
\begin{aligned}
-\frac{\beta}{\frac{B_{i}+\theta}{\tau} r_{i}+W_{i}}+\lambda+\kappa_{i}-\nu_{i} & =0 ; \\
\lambda\left(\sum_{i=1}^{N} W_{i}-W\right) & =0 ; \\
\kappa_{i}\left(W_{i}-W\right) & =0 ; \forall i=1, \ldots, N . \\
\nu_{i} W_{i} & =0 ; \forall i=1, \ldots, N . \\
0 \leq W_{i} & \leq W ; \forall i=1, \ldots, N . \\
\sum_{i=1}^{N} W_{i} & =W ; \\
\lambda \geq 0, \kappa_{i} \geq 0, \nu_{i} & \geq 0 ; \forall i=1, \ldots, N .(42)
\end{aligned}
$$

By solving the KKT conditions above, the optimal solution for $W_{i}$ is:

$$
W_{i}^{\star}\left(r_{i}(\lambda)\right)=\min \left(W, \max \left(0, \frac{\beta}{\lambda}-\frac{B_{i}+\theta}{\tau} r_{i}(\lambda)\right)\right),
$$

where $\sum_{i=1}^{N} \min \left(W, \max \left(0, \frac{\beta}{\lambda}-\frac{B_{i}+\theta}{\tau} r_{i}(\lambda)\right)\right)=W$.

Then, we substitute $W_{i}^{\star}\left(r_{i}(\lambda)\right)$ back to (41) and the Lagrangian becomes

$$
L\left(W_{i}^{\star}\left(r_{i}(\lambda)\right), r_{i}, \lambda, \kappa_{i}, \nu_{i}\right)=-\sum_{i=1}^{N} f\left(r_{i}, \lambda\right)+\lambda W,
$$

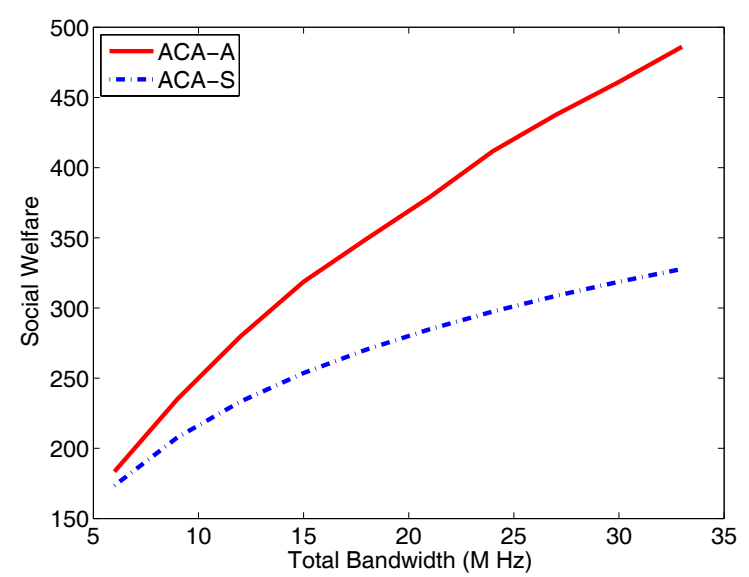

Fig. 3. The social welfare comparison between ACA-A and ACA-S algorithms.

where $f\left(r_{i}, \lambda\right)$ is defined in $(21)$.

Therefore, the optimal $r_{i}^{\star}$ is

$$
r_{i}^{\star}=\arg \max _{r_{i}} f\left(r_{i}, \lambda\right) .
$$

In all, the solution to the optimization problem in (39) is

$$
\left\{\begin{array}{l}
r_{i}^{\star}=\arg \max _{r_{i}} f\left(r_{i}, \lambda\right), \\
W_{i}^{\star}=\min \left(W, \max \left(0, \frac{\beta}{\lambda}-\frac{B_{i}+\theta}{\tau} r_{i}^{\star}\right)\right),
\end{array}\right.
$$

where $\lambda$ is the constant that satisfies $\sum_{i=1}^{N} \min \left(W, \max \left(0, \frac{\beta}{\lambda}-\frac{B_{i}+\theta}{\tau} r_{i}^{\star}(\lambda)\right)\right)=W$.

Thus, $\left(W_{1}^{\star}, r_{1}^{\star}, \ldots, W_{N}^{\star}, r_{N}^{\star}\right)$ is the solution that maximizes the social welfare.

Theorem 7: Compared with ACA-A, ACA-S achieves a smaller, if not equal, social welfare.

Proof: According to Algorithm 1 and 3, we can see that if we constrain $W_{i}$ to be 0 or $W$, i.e. $W_{i} \in\{0, W\}$, then ACA-A becomes ACA-S. Therefore, ACA-S is a special case of ACA-A with constraint $W_{i} \in\{0, W\}$.

Since the feasible set of ACA-S is a subset of that of ACA$A$, the social welfare of ACA-S is smaller than, if not equal to, that of ACA-A algorithm.

\section{Remarks}

From the previous three subsections, we can see that all three schemes (ACA-S, ACA-T, and ACA-A) converge in a finite number of clocks. We also find that ACA-S and ACA$A$ are cheat-proof while ACA-T is not. In terms of social welfare, we show that ACA-T and ACA-A can maximize the social welfare while ACA-S may not. Therefore, ACA-A is a good solution to multimedia cognitive radio networks since it can achieve maximal social welfare in a cheat-proof way.

\section{Simulation Results}

In order to evaluate the proposed spectrum allocation schemes, we conduct simulation on real video data. Five video sequences: Akiyo, Carphone, Foreman, Football, and Mobile in QCIF format, are tested. Notice that these video sequences include slow, medium or fast motion, and smooth or complex scene. We use the state-of-art scalable video codec (JSVM 


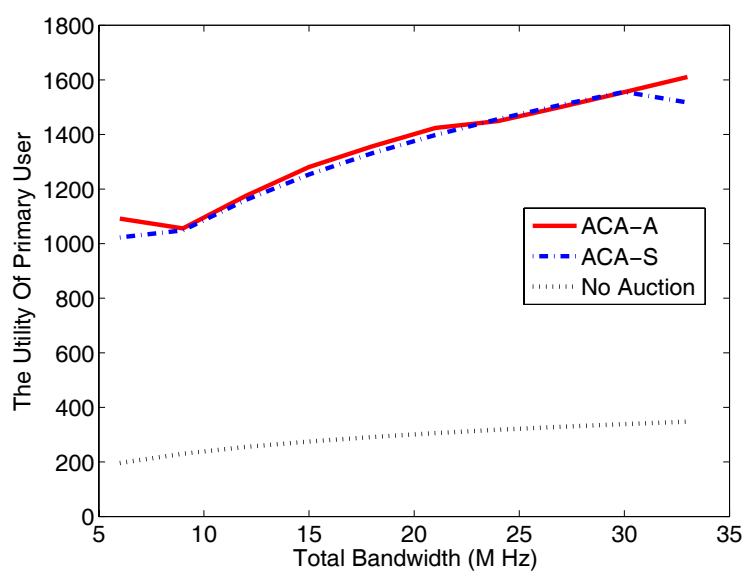

Fig. 4. The primary user's utility comparison among different methods.

9.17) to encode the video sequences [28]. By utilizing the SNR scalability, we compress each video sequence at three different quality layers.

We first evaluate the cheat-proof performance of ACA-T and ACA-A. We assume that the SU $u_{3}$ who transmits Foreman will cheat while other users are honest. We assume that $u_{3}$ reports a false demand $\tilde{W}_{3}^{t}$ by scaling the optimal demand $W_{3}^{t}$ with a factor $k$, i.e. $\tilde{W}_{3}^{t}=\min \left(W, \max \left(0, k W_{3}^{t}\right)\right)$. As shown in Fig. 2, we can see that with ACA-T, $u_{3}$ achieves the maximal utility when $k$ is around 0.7 . Therefore, all SUs have the incentive to report a smaller demand at every clock. In this case, the auction will conclude at a lower price. Thus, ACA-T is not cheat-proof. However, with ACA-A, we can see that $u_{3}$ achieves the maximal utility when $k=1$, which means that no SUs have the incentive to cheat since any cheating will lead to a lower utility. This simulation result verifies Theorem 4 and Theorem 5.

Then, we compare ACA-A with ACA-S in terms of social welfare. The results are shown in Fig. 3. We can see that ACAA achieves a much higher social welfare compared with ACA$\mathrm{S}$. This is because with ACA-S, each SU can only choose to utilize the whole spectrum or not to utilize the spectrum. However, with ACA-A, each SU has the chance to utilize a fraction of the entire spectrum. This simulation result verifies

\section{Theorem 7.}

Third, we compare ACA-A with ACA-S in terms of PU's utility. The results are shown in Fig. 4. We can see that ACAA and ACA-S have the similar performance in terms of PU's utility. We also find that both ACA-A and ACA-S achieve much better performance compared with "no auction" where PU always chooses to self-utilize the spectrum.

Finally, we compare the proposed approach with the VCGbased approach in [19] in terms of the cheat-proof performance of the primary user. Note that in [19], the primary user computes each transfer $\kappa_{i}$ for the secondary user $i$ using

$\kappa_{i}=\sum_{k \neq i} U_{k}\left(r_{k}^{\star}, B_{k}, W_{k}^{\star}\right)-\rho\left[\max _{r_{k}, W_{k}} \sum_{k \neq i} U_{k}\left(r_{k}, B_{k}, W_{k}\right)\right]$.

with $\rho=1$.

We find that, for any fixed $\rho$, the approach in [19] can enforce every secondary user to report true information. The

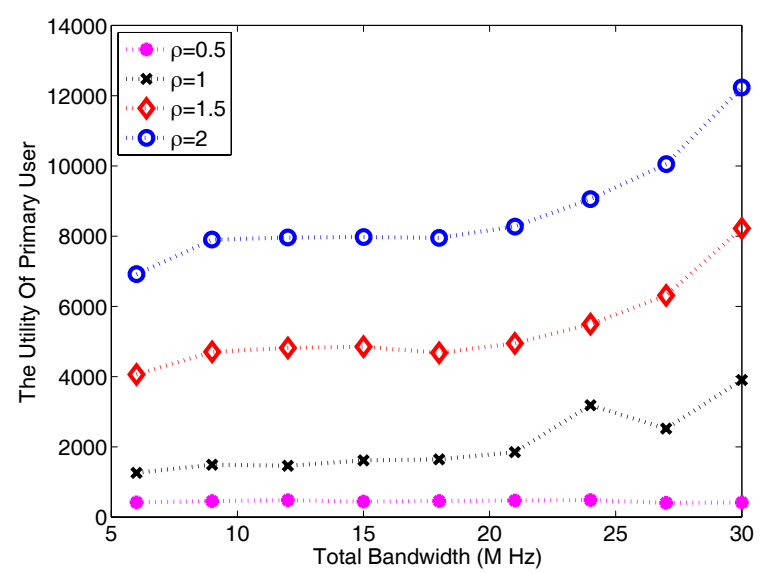

Fig. 5. The primary user's utility with different $\rho$ for the VCG-based approach in [19].

proof is the same as the proof of Proposition 3 in [19]. Then, in Fig. 5, we show the PU's utility with different $\rho$ for the VCG-based approach in [19]. We can see that PU's utility increases as $\rho$ increase. Since the objective of the primary user is to maximize his/her revenue, i.e. maximizing $-\sum_{i} \kappa_{i}$, he/she has the incentive to use a larger $\rho$ to achieve a larger revenue. Therefore, the VCG-based approach in [19] is not cheat-proof to the primary user. On the other hand, in our proposed clock auction scheme, since both the primary user and the secondary users know the unit price and the amount of the allocated bandwidth at every clock, the primary user cannot cheat. Therefore, our approach can achieve comparable social welfare to that in [19], while cheat-proof to both the primary user and secondary users.

\section{CONCLUSION}

In this paper, we investigate the problem of multimedia streaming over cognitive radio networks and propose three auction-based schemes (ACA-S, ACA-T, and ACA-A) to distributively allocate the spectrum. We prove and demonstrate with simulation results that ACA-T and ACA-A can efficiently allocate the spectrum and achieve maximal social welfare. We also prove and verify with simulations that ACA-S and ACA-A are cheat-proof and can enforce the selfish secondary users to report their true demands at every clock. Moreover, with the proposed schemes, the primary user and secondary users can seamlessly switch among different quality levels since the uniquely scalable and delay-sensitive characteristics of multimedia data and the resulting impact on users' viewing experiences of multimedia content are explicitly considered in the utility functions.

\section{REFERENCES}

[1] Federal Communications Commission, "Facilitating opportunities for flexible, efficient and reliable spectrum use employing cognitive radio technologies: notice of proposed rule making and order," FCC Document ET Docket No. 03-108, Dec. 2003.

[2] S. Haykin, "Cognitive radio: brain-empowered wireless communications," IEEE J. Sel. Areas Commun., vol. 23, no. 2, pp. 201-220, Feb. 2005.

[3] L. Cao and H. Zheng, "Distributed spectrum allocation via local bargaining," in Proc. IEEE SECON, Sep. 2005, pp. 475-486. 
[4] Z. Han, C. Pandana, and K. J. R. Liu, "Distributive opportunistic spectrum access for cognitive radio using correlated equilibrium and no-regret learning," in Proc. IEEE WCNC, Mar. 2007, pp. 11-15.

[5] R. Etkin, A. Parekh, and D. Tse, "Spectrum sharing for unlicensed bands," IEEE J. Sel. Areas Commun., vol. 25, no. 3, pp. 517-528, Apr. 2007.

[6] Y. Wu, B. Wang, K. J. R. Liu, and T. C. Clancy, "Repeated open spectrum sharing game with cheat-proof strategies," IEEE Trans. Wireless Commun., vol. 8, no. 4, pp. 1922-1933, Apr. 2009.

[7] Z. Ji and K. J. R. Liu, "Dynamic spectrum sharing: a game theoretical overview," IEEE Commun. Mag., vol. 45, no. 5, pp. 88-94, May 2007.

[8] S. Gandhi, C. Buragohain, L. Cao, H. Zheng, and S. Suri, "A general framework for wireless spectrum auctions," in Proc. IEEE DySPAN, Apr. 2007, pp. 22-33.

[9] O. Ileri, D. Samardzija, and N. B. Mandayam, "Demand responsive pricing and competitive spectrum allocation via a spectrum server," in Proc. IEEE DySPAN, Nov. 2005, pp. 194-202.

[10] J. Huang, R. Berry, and M. L. Honig, "Auction-based spectrum sharing," ACM Mobile Netw. Applicat. (MONET), vol. 11, pp. 405-418, June 2006.

[11] Z. Ji and K. J. R. Liu, "Belief-assisted pricing for dynamic spectrum allocation in wireless networks with selfish users," in Proc. IEEE SECON, Sep. 2006, pp. 119-127.

[12] Y. Wu, B. Wang, K. J. R. Liu, and T. Clancy, "A scalable collusionresistant multi-winner cognitive spectrum auction game," IEEE Trans. Commun., vol. 57, no. 12, pp. 3805-3816, Dec. 2009.

[13] X. Zhou, S. Gandi, S. Suri, and H. Zheng, "eBay in the sky: strategyproof wireless spectrum auctions," in Proc. MobiCom, Sep. 2008, pp. $2-13$.

[14] Z. Ji and K. J. R. Liu, "Multi-stage pricing game for collusion-resistant dynamic spectrum allocation," IEEE J. Sel. Areas Commun., vol. 26, no. 1, pp. 182-191, Jan. 2008

[15] B. Wang, Y. Wu, Z. Ji, K. J. R. Liu, and T. C. Clancy, "Game theoretical mechanism design methods: suppressing cheating in cognitive radio networks," IEEE Signal Process. Mag., vol. 25, no. 6, pp. 74-84, Nov. 2008.

[16] H. P. Schang and M. van der Schaar, "Queuing-based dynamic channel selection for heterogeneous multimedia applications over cognitive radio networks," IEEE Trans. Multimedia, vol. 10, no. 5, pp. 896-909, Aug. 2008.

[17] S. Ali and R. Yu, "Cross-layer QoS provisioning for multimedia transmissions in cognitive radio networks," in Proc. IEEE WCNC, 2009.

[18] H. Kushwaha, Y. Xing, R. Chandramouli, and H. Heffes, "Reliable multimedia transmission over cognitive radio networks using fountain codes," Proc. IEEE, vol. 96, no. 1, pp. 155-165, Jan. 2008.

[19] A. Fattahi, F. Fu, M. van der Schaar, and F. Paganini, "Mechanismbased resource allocation for multimedia transmission over spectrum agile wireless networks," IEEE J. Sel. Areas Commun., vol. 25, no. 3, pp. 601-612, Apr. 2007

[20] V. Krishna, Auction Theory. Academic Press, 2002.

[21] L. M. Ausubel, "An efficient ascending-bid auction for multiple objects," American Economic Review, vol. 94, pp. 1452-1475, 2004.

[22] C. U. Saraydar, N. B. Mandayam, and D. J. Goodman, "Pareto efficiency of pricing-based power control in wireless data networks," in Proc. IEEE WCNC, 1999, pp. 231-235.

[23] P. Golle, K. Leyton-Brown, and I. Mironov, "Incentives for sharing in peer-to-peer networks," in Proc. ACM Conf. Electronic Commerce, 2001, pp. 264-267.

[24] P. Marbach and R. Berry, "Downlink resource allocation and pricing for wireless networks," in Proc. IEEE INFOCOM, 2002, pp. 1470-1479.

[25] J. Huang, Z. Ji, M. Chiang, and A. K. Katsaggelos, "Joint source adaptation and resource allocation for multi-user wireless video streaming," IEEE Trans. Circuits Syst. Video Technol., vol. 18, no. 5, pp. 582-595, May 2008.

[26] D. Palomar and M. Chiang, "A tutorial on decomposition methods for network utility maximization," IEEE J. Sel. Areas Commun., vol. 24, no. 8, pp. 1439-1451, Aug. 2006.

[27] S. Boyd and L. Vandenberghe, Convex Optimization. Cambridge University Press, 2004

[28] [Online]. Available: http://ip.hhi.de/imagecom_G1/savce/downloads/SVCReference-Software.htm

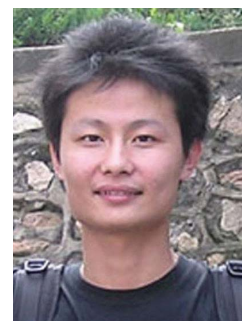

Yan Chen (S'06) received the bachelor's degree from the University of Science and Technology of China (USTC) in 2004 and the M.Phil degree from Hong Kong University of Science and Technology (HKUST) in 2007. Currently, he is a PhD student in the Department of Electrical and Computer Engineering at the University of Maryland, College Park.

His current research interests are in game theoretical modelling for multimedia social networks, multimedia signal processing, cooperative multimedia communication and networking, and multimedia forensic. He received the University of Maryland Future Faculty Fellowship in 2010 .

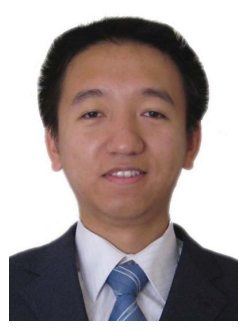

Yongle Wu (S'08) received the B.S. (with highest honor) and M.S. degrees in electronic engineering from Tsinghua University, Beijing, China, in 2003 and 2006, respectively. He is currently working towards the Ph.D. degree in the Department of Electrical and Computer Engineering, University of Maryland, College Park.

His current research interests are in the areas of wireless communications and networks, including cognitive radio techniques, dynamic spectrum access, and network security. Mr. Wu received the Graduate School Fellowship from the University of Maryland in 2006, and the Future Faculty Fellowship from the A. James Clark School of Engineering, University of Maryland in 2009.

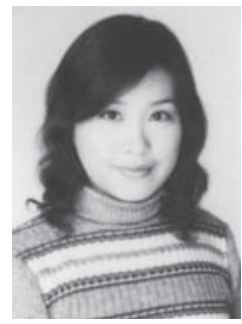

Beibei Wang (S'07) received the B.S. degree in electrical engineering (with the highest honor) from the University of Science and Technology of China, Hefei, in 2004, and the Ph.D. degree in electrical engineering from the University of Maryland, College Park, in 2009. She is currently a research associate at the University of Maryland.

Her research interests include dynamic spectrum allocation and management in cognitive radio systems, wireless communications and networking, game theory, wireless multimedia, and network security. Dr. Wang was the recipient of the Graduate School Fellowship, the Future Faculty Fellowship, and the Dean's Doctoral Research Award from the University of Maryland.

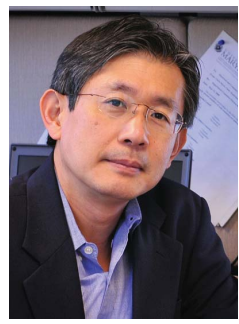

K. J. Ray Liu (F'03) was named a Distinguished Scholar-Teacher at the University of Maryland, College Park, in 2007. He is the Associate Chair of Graduate Studies and Research in the Electrical and Computer Engineering Department and leads the Maryland Signals and Information Group, conducting research encompassing broad aspects of wireless communications and networking, information forensics and security, multimedia signal processing, and biomedical engineering.

Dr. Liu is the recipient of numerous honors and awards including the IEEE Signal Processing Society Technical Achievement Award and Distinguished Lecturer. He also received various teaching and research recognitions from the University of Maryland including the universitylevel Invention of the Year Award; and the Poole and Kent Senior Faculty Teaching Award and Outstanding Faculty Research Award, both from the A. James Clark School of Engineering. Dr. Liu is a Fellow of IEEE and AAAS.

Dr. Liu is President-Elect and was Vice President - Publications of the IEEE Signal Processing Society. He was the Editor-in-Chief of IEEE Signal Processing Magazine and the founding Editor-in-Chief of the EURASIP Journal on Advances in Signal Processing.

His recent books include Cognitive Radio Networking and Security: A Game Theoretical View, Cambridge University Press, 2010; Cooperative Communications and Networking, Cambridge University Press, 2008; Resource Allocation for Wireless Networks: Basics, Techniques, and Applications, Cambridge University Press, 2008; Ultra-Wideband Communication Systems: The Multiband OFDM Approach, IEEE-Wiley, 2007; Network-Aware Security for Group Communications, Springer, 2007; Multimedia Fingerprinting Forensics for Traitor Tracing, Hindawi, 2005; and the Handbook on Array Processing and Sensor Network, IEEE-Wiley, 2009. 